\title{
Evaluating the load-carrying ability of compressed reinforced concrete elements with heterogeneous strength properties of concrete
}

\author{
Alexander Pischulev ${ }^{1}$, Denis Panfilov ${ }^{1, *}$, and Andrey Aleshin ${ }^{1}$ \\ ${ }^{1}$ Samara State Technical University, Institute of Architecture and Civil Engineering, \\ 194, Molodogvardeyskaya St, 443001, Samara, Russia
}

\begin{abstract}
This article presents a methodology of determining the loadcarrying ability of compressed reinforced concrete elements with layers of various concrete strength and describes the stress-strain behaviour of these elements. The methodology is based on the use of a deformation model and diagrams of materials deformation [1,2]. The authors used this methodology to develop an algorithm. The algorithm makes it possible to evaluate stress and strain state of normal sections at all stages of loading up to destruction. The solution of the problem is shown as a data table. Analysis of this data table allows to estimate an expected pattern of the samples destruction. The paper also gives an example of calculation of a compressed reinforced concrete element with heterogeneous strength properties of a compressed area with symmetrical reinforcement (4 bars). The calculation methodology in this article has been successfully used to evaluate the load bearing capacity of the described samples and those of other authors.
\end{abstract}

\section{Introduction}

Inspections and examinations of buildings and other structures revealed that reinforced concrete elements with different strength of concrete layers are quite frequent within these constructions. The shift in the strength of concrete layers may be caused by technological factors or by mechanical or corrosive damage. The methods currently available to determine the depth of corrosion damage and residual mechanical strength characteristics of concrete are generally based on a review of corrosion of materials over time, with the effect of a certain reagent based on laboratory research. However, the operating conditions of real structures are often different from laboratory conditions, since the structure may be susceptible to the aggressive effects of several reagents, and the actual temperaturehumidity conditions may differ from those based on calculations. Errors in corrosion processes prediction in reinforced concrete structures may reduce the accuracy of calculations and, in some cases, lead to reassessing their load bearing capacity. The question of calculation of structures with disrupted strength of concrete is very actual and it has been the center of analysis in in recent years by many Russian and foreign researchers.

* Corresponding author: panda-w800i@yandex.ru 
Theoretical and experimental research in this area (6-9) is currently being carried out in the Department of Building Structures of Samara State Technical University. The authors of the research suggest that load bearing capacity capacity of disrupted structures should be assessed by means of calculation algorithms using non-linear diagrams of concrete and reinforcement deformation.

\section{Materials and Methods}

Modern methods for estimating mechanical strength characteristics of concrete [3-5] allow to estimate mechanical strength characteristics of concrete in the section with high accuracy. By analyzing strength properties of concrete, relatively homogeneous concrete areas can be identified in the section under investigation. Once data on strength characteristics of the relatively homogeneous concrete areas are obtained, coefficients $a_{c}$ and $b_{c}$ are defined for the exponential function 1 proposed by professor G.V. Murashkin [1]

$$
\sigma_{b}(\varepsilon)=a_{c} \cdot \varepsilon^{b_{c}} \cdot \exp \left(\frac{-b_{c} \cdot \varepsilon}{p}\right),
$$

where $\sigma_{b}$ is the stress in the concrete; $\varepsilon$-relative deformation of concrete; ac и bc $-a_{c}$ and $b_{c}$ - coefficients of exponential function $f(\varepsilon)=\varepsilon \cdot E$, which describes shift in the elasticity module from the magnitude $\varepsilon ; p$ - relative deformation of concrete in the chart of the maximum value of the stresses.

The deformation model of the elements shown in Figure 1 is accepted for calculation.

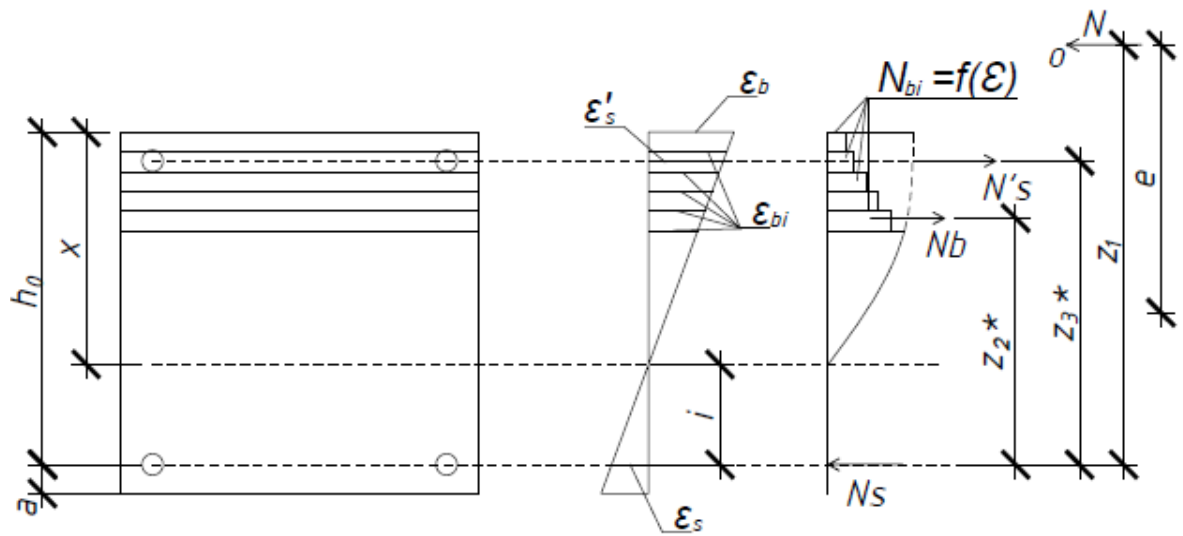

Fig. 1. Diagram of the calculation model of off-centered compressed reinforced concrete element with heterogeneous strength properties of the concrete at the height of the section. $N_{b}$-force by the concrete of the compressed section area; $N_{b i}$ - force taken by the concrete of the relatively chosen layer; $\varepsilon_{b i}$ - relative deformation of concrete in the most compressed part of the section; $\varepsilon_{s}$-relative deformation of reinforcement; $N_{s}$ - is the force of reinforcement.

\section{Results and Discussion}

Deformation of concrete in the most compressed concrete zone can be calculated by equation of equilibrium of internal and external forces is formed 2).

$$
N=N_{s}^{\prime}+N_{b}-N_{s},
$$


where $N_{s}, N_{s}^{\prime}-$ a force taken, respectively, by stretched and compressed reinforcement; $N_{b}$ - a force taken by compressed concrete.

Force $N_{s}$ is determined according to the level of deformation in reinforcement. If reinforcement deformations have not reached the yield limit, force $N_{s}$ is determined by the formula (3), otherwise by the formula (4).

$$
N_{s}=A_{s} \cdot \sigma_{s}\left(\varepsilon_{s}\right)
$$

where $A_{s}$ the cross-sectional area of reinforcement; $\sigma_{s}\left(\varepsilon_{s}\right)$ - a function of stresses in reinforcement from deformation; $\varepsilon_{\mathrm{s}}$-relative deformation at the center of gravity level of the section of tensile reinforcement.

$$
N_{s}=A_{s} \cdot R_{s},
$$

where $A_{s}$ - the cross-sectional area of reinforcement; $R_{s}$ - designed resistance of reinforcement to axial stretch;

Force $N_{s}^{\prime}$ is defined in a similar way By formula (5), (6).

$$
N_{s}^{\prime}=A_{s}^{\prime} \cdot \sigma_{s}\left(\varepsilon_{s}^{\prime}\right)
$$

where $A_{s}^{\prime}$ - the cross-sectional area of compressed reinforcement; $\sigma_{s}\left(\varepsilon_{s}{ }^{\prime}\right)$ - a function of stresses in reinforcement from deformation; $\varepsilon_{s}$ - relative deformations at the center of gravity level of the section of compressed reinforcement.

$$
N_{s}^{\prime}=A_{s}^{\prime} \cdot R_{s c}
$$

where $A_{s}{ }^{\prime}$ - the cross-sectional area of compressed reinforcement; $R_{s c}$ - designed resistance of reinforcement to compression;

Force of $N_{b}$ is determined as a sum of the forces (7) taken by concrete of relatively homogeneous layers:

$$
N_{b}=\sum_{i=1}^{n} N_{b i},
$$

where $N_{b i}-$ a force taken by compressed concrete of layer $i$ by formula (8).

$$
N_{b i}=b \cdot \int_{m i}^{n i} \sigma_{b i}(x) d x
$$

where $b$ - width of the rectangular section of the bending element; $\sigma_{b}(x)$ - function of shift in stresses in concrete (9); $m_{i}$ and $n_{i}-$ are the lower and upper limits of integration corresponding to the lower and upper boundaries of the corresponding selected layers in the compressed section area.

$$
\sigma_{b}(x)=a_{c} \cdot\left(\frac{\varepsilon_{b} \cdot x}{k}\right)^{b_{c}} \cdot \exp \left(\frac{-b_{c} \cdot\left(\varepsilon_{b} \cdot \frac{x}{k}\right)}{p}\right),
$$


where $\sigma_{b}-$ the stress in the concrete; $\varepsilon_{b}-$ relative deformation of the most compressed face of the element resulting from the reparsing; $x$-the coordinate of the compressed concrete zone point in question; ; $k$ - the full height of the compressed concrete area resulting from the reparsing; $a_{c}$ and $b_{c}$ - coefficients of exponential function $f(\varepsilon)=\varepsilon \cdot E$, which describes the shift of the elasticity module from magnitude $\varepsilon ; p$ - relative deformation of concrete in the chart $\mathrm{f}$ the maximum value of the stresses.

Next we define the eccentricity $e$ from equation (10):

$$
N_{s}^{\prime}(e-h / 2+a)+N_{b}\left(e+h / 2-a-S_{b} / A_{b}-i\right)-N_{s}(e+h / 2-a)=0
$$

The equation of moments of external and internal forces is drawn relative to the center of gravity of the section of tensile reinforcement (11):

$$
\sum M=0: N_{b}\left(\frac{S_{b}}{A_{b}}+i\right)+N_{s}^{\prime}\left(h_{0}-a^{\prime}\right)-N(e+h / 2-a)=0
$$

where $Z_{2}{ }^{*}-$ a shoulder of $\mathrm{N}_{\mathrm{b}}$ force as regard to the center of gravity of stretched reinforcement; $\quad Z_{3}{ }^{*}$ - a shoulder of effort $N_{s}$ as regard to the center of gravity of tensile reinforcement; $Z_{1}$ - a shoulder of $N$ force as regard to the center of gravity of stretched reinforcement.

To determine the distance between the center of gravity of stretched reinforcement and the application point of the resultant force $\mathrm{N}_{\mathrm{b}}$ we first found a static moment of concrete stress diagram and its area.

The area of the diagram of a compressed area of concrete is determined according to formula (12):

$$
A_{b}=\sum_{i=1}^{n} A_{b i}
$$

where $A_{b i}$ - the area of a diagram of the i-th layer determined by formula (13).

$$
A_{b i}=\int_{m i}^{n i} \sigma_{b i}(x) d x
$$

where $\sigma_{b}(x)$ is the function of changing stresses in concrete (7); $m_{i}$ and $n_{i}-$ are the lower and upper limits of integration corresponding to the lower and upper bounds of the corresponding selected layers in the compressed section area.

The static moment of the diagram of a compressed area of concrete is determined according to formula (14):

$$
S_{b}=\sum_{i=1}^{n} S_{b i}
$$

where $A_{b i}$ - the area of a diagram of the i-th layer determined by formula (1).

$$
S_{b i}=\int_{m i}^{n i} \sigma_{b i}(x) x d x
$$


Then the shoulder of force $\mathrm{N}_{\mathrm{b}}$ is to be calculated by formula (16):

$$
Z_{2}^{\prime}=i+\frac{S_{b}}{A_{b}}
$$

The calculation algorithm is given below.

The basic data are: $b, h$ - an actual width and height of the calculation section; $h_{0}-$ working height of the section; $a_{c j}, b_{c j}$ - coefficients of analytical dependence describing stress and strain state of concrete with relatively homogeneous layer; $P_{j}-$ relative deformation of concrete corresponding to the stress in concrete of equal to $R_{b}$ in a separately chosen layer; $E_{b j}-$ a starting module of concrete elasticity; $A_{s}, R_{s}, E_{s}$ - area, design resistance, and elasticity module of working reinforcement; shoulders $Z_{1}, Z_{2}{ }^{*}, Z_{3}{ }^{*}$, external force moment $M_{i}$, force in concrete $N_{b}$ and reinforcement $N_{s}$ and $N_{s}$.

Cycle 1. During this cycle, the values are set sequentially from 0 to 0.0055 , to the value of $\varepsilon_{b, \max }$, where ( $\varepsilon_{b, \max }$ - deformation of concrete at the most compressed part.)

Cycle 2 (a cycle in Cycle 1). During this cycle, we successively assign to the variable $\mathrm{i}$ values from -100 to $h_{0}$, где $\mathrm{i}$, where $i$ is the distance from the center of gravity of the section of stretched reinforcement to the zero line.

Altitude of compressive zone of concrete is determined by the formula: $X_{i}=h_{0^{-}}-i$

By assigning soma value to the values $I$ and $\varepsilon_{b, \max }$ determine deformations:

- in compressed reinforcement: $\varepsilon_{s}^{{ }^{\prime}}=\varepsilon_{b, \max }\left(h_{0}-i-a^{\prime}\right) /\left(h_{0}-i\right)$

- in stretched (least compressed) reinforcement: $\varepsilon_{s}=\left(\varepsilon_{b, \max }-i\right) /\left(h_{0}-i\right)$.

Determination of $N_{s}$ force: if $0 \leq \varepsilon_{s} \leq R_{s} / E_{s}$, then $N_{s}=E_{s} \cdot \varepsilon_{s} \cdot A_{s}$, otherwise $N_{s}=A_{s} \cdot R_{s}$.

Determination of $N_{s}$ ' force: if $0 \leq \varepsilon_{s} \leq R_{s c} / E_{s}$, then $N_{s}{ }^{\prime}=E_{s} \cdot \varepsilon_{s}{ }^{\prime} \cdot A_{s}{ }^{\prime}$, otherwise $N_{s}{ }^{\prime}=A_{s}{ }^{\prime} \cdot R_{s c}$.

Determination of the number of layers $i$, that are in the compressed section area.

Determination of $N_{b}$ force by formula (8).

Determination of $N$ force $N=N_{s}{ }^{\prime}+N_{b}-N_{s}$

Eccentricity $e$ can be defined from equation (10).

The condition of breaking cycle 2 is calculated by an equation (11).

Then intermediate values are calculated, which are the solution of internal forces equation with some value of $\varepsilon_{b, \max }$, which goes as follows:

1. The area of the diagram of a compressed area of concrete is determined according to equation (12):

2. The static moment of the diagram of a compressed area of concrete is determined according to equation (14):

3. $N$-force eccentricity is : $e$

4. Force $N$

5. Shoulders $Z_{1} ; Z_{2}{ }^{*} ; Z_{3} *$

6. Moment of external force $M_{i}=N \cdot e$.

7. Force in concrete $N_{b}$ and reinforcement $N_{s}$ and $N_{s}$ '.

8. The values of relative deformations in the most compressed face of concrete $\varepsilon_{b i}$ and at the center of gravity of the cross-section of stretched and compressed reinforcement $\varepsilon_{s i}$ and $\varepsilon_{s i}$.

Interruption of Cycle 1 occurs when the following inequality is performed: $M_{i}>M_{i}+1$.

The results of the calculations are presented as a table of values for all intermediate solutions. 


\section{Conclusions}

The paper provides a methodology for determining the capacity of compressed reinforced concrete elements, including off-centered compressed elements, with varying strengths of concrete layers based on the deformation model and non-linear material deformation diagrams (concrete and steel). The paper also introduces a step-by-step algorithm for estimating stress and strain state of compressed reinforced concrete elements with symmetrical reinforcement, with the possibility of taking into account the heterogeneous strength properties of concrete in a compressed zone. The solution of the problem is shown as a data table. Analysis of this data table allows to estimate an expected pattern of the samples destruction.

The results of this scientific research are recommended for practical application in design organizations and can be used to improve existing regulatory documents for calculating reinforced concrete elements.

\section{References}

1. G.V. Murashkin, V.G. Murashkin, News of Higher Educational Institutions. Construction 10, 4-6 (1997)

2. D.A. Panfilov, A.A. Pishchulev, K.I. Gimadetdinov, Industrial and civil engineering 3, 80-84 (2014)

3. V.O. Krichke, G.V. Murashkin, Yu.V. Volkov, D.V. Goryainov, RF Patent 2179722. Means of analysing structure and strength of concrete in the process of sampling from concrete structures by drilling, Ann. 6 March 2000 (Published 20.02.2002)

4. V.O. Krichke, G.V. Murashkin, Yu.V. Volkov, RF Patent 2198399. Information system for analysing structure and strength of concrete in the process of sampling from concrete structures by drilling, Ann.10 March 2000 (Published 10.02.2003)

5. V.B. Gavrilov, Assessment of mechanical and structural characteristics of concrete in building constructions by a method of local destruction (2004).

6. Valery B. Filatov, Alexander A. Suvorov, Procedia Engineering 153, 144-150 (2016)

7. E.M. Rudakov, A.E. Frolov, Urban Construction and Architecture 3, 98-102 (2013).

8. A.N. Ilyin, D.A. Panfilov, D.V. Litvinov, P.N. Slavkin, Urban Construction and Architecture 1, 82-89 (2015). 